\title{
Reabilitação fonoaudiológica do paciente com COVID-19: Uma revisão integrativa
}

\author{
Phonoaudiological rehabilitation of the patient with COVID-19: An integrative review \\ Rehabilitación fonoaudiológica del paciente con COVID-19: Una revisión integrativa
}

Recebido: 14/06/2021 | Revisado: 19/06/2021 | Aceito: 22/06/2021 | Publicado: 08/07/2021

Talyssa Sandes de Sena

ORCID: https://orcid.org/0000-0002-9501-8793 Faculdade de Ensino Superior do Piauí, Brasil E-mail: talyssasena@gmail.com

Gislene Mariana Pereira Castelo Branco

ORCID: https://orcid.org/0000-0003-4393-771X Faculdade de Ensino Superior do Piaú, Brasil E-mail: gi.marianna@hotmail.com

Ruth Raquel Soares de Farias

ORCID: https://orcid.org/0000-0002-0988-0900

Faculdade de Ensino Superior do Piaú, Brasil E-mail: ruthraquelsf@gmail.com

\begin{abstract}
Resumo
A COVID-19 é uma doença do trato respiratório superior, os casos mais graves da doença frequentemente necessitam de terapia intensiva e apresentam síndrome de desconforto respiratório agudo (SDRA), necessitando de intubação orotraqueal prolongada e/ou reintubação. O desenvolvimento de complicações pós intubação são muito comuns e influenciam a atividade dos músculos relacionados à deglutição. Neste sentido, a atuação do fonoaudiólogo na equipe multiprofissional do paciente com COVID-19 é de grande importância, especialmente na reabilitação pós terapia intensiva. Objetivo: revisar a literatura disponível sobre a da atuação do fonoaudiólogo em pacientes com COVID-19 submetidos à terapia intensiva. Método: Trata-se de uma revisão integrativa da literatura realizada nas bases de dados Bireme, Scielo, Science Direct, e PubMed, incluindo estudos publicados nos anos de 2019 e 2021. Resultados: Foi possível verificar que a atuação do fonoaudiólogo é essencial, através da avaliação e intervenção fonoaudiológica a fim de evitar que o quadro se agrave ainda mais. Os estudos demonstram que o fonoaudiólogo atua principalmente nos distúrbios de deglutição e da voz. Conclusão: O fonoaudiólogo é um profissional indispensável no tratamento de pacientes com COVID-19, sobretudo os pacientes submetidos à intubação orotraqueal, pois através da sua intervenção terapêutica, facilita o trabalho dos demais profissionais da equipe multidisciplinar e possibilita mais qualidade de vida ao paciente.
\end{abstract}

Palavras-chave: Fonoaudiologia; Transtornos de deglutição; Disfonia; COVID-19; Unidades de terapia intensiva.

\begin{abstract}
COVID-19 is a disease of the upper respiratory tract, the most severe cases of the disease often require intensive therapy and have acute respiratory distress syndrome (ARDS), requiring prolonged orotracheal intubation and / or reintubation. The development of post-intubation complications is very common and influences the activity of muscles related to swallowing. In this sense, the role of the speech therapist in the multidisciplinary team of patients with COVID-19 is of great importance, especially in rehabilitation after intensive care. Objective: review the available literature on the performance of the speech therapist in patients with COVID-19 undergoing intensive care. Method: This is an integrative literature review carried out in the databases Bireme, Scielo, Science Direct, and PubMed, including studies published in the years 2019 and 2020. Results: It was possible to verify that the performance of the speech therapist it is essential, through the evaluation and speech therapy intervention in order to prevent the condition from getting worse. Studies show that the speech therapist works mainly in swallowing and voice disorders. Conclusion: The speech therapist is an indispensable professional in the treatment of patients with COVID-19, especially patients undergoing orotracheal intubation, because through their therapeutic intervention, it facilitates the work of the other professionals of the multidisciplinary team and enables better quality of life patient.
\end{abstract}

Keywords: Speech therapy; Deglutition disorders; Dysphonia; COVID-19; Intensive care units. 
Research, Society and Development, v. 10, n. 8, e13610817154, 2021

(CC BY 4.0) | ISSN 2525-3409 | DOI: http://dx.doi.org/10.33448/rsd-v10i8.17154

\begin{abstract}
Resumen
COVID-19 es una enfermedad del tracto respiratorio superior, los casos más graves de la enfermedad a menudo requieren terapia intensiva y tienen síndrome de dificultad respiratoria aguda (SDRA), que requiere intubación orotraqueal prolongada y / o reintubación. El desarrollo de complicaciones postintubación es muy común e influye en la actividad de los músculos relacionados con la deglución. En este sentido, el papel del logopeda en el equipo multiprofesional del paciente con COVID-19 es de gran importancia, especialmente en la rehabilitación después de cuidados intensivos. Objetivo: revisar la literatura disponible sobre el desempeño del logopeda en pacientes con COVID-19 en cuidados intensivos. Método: Se trata de una revisión integradora de la literatura realizada en las bases de datos Bireme, Scielo, Science Direct y PubMed, incluyendo estudios publicados en los años 2019 y 2020. Resultados: Se pudo constatar que la actuación del logopeda es fundamental, a través de la evaluación e intervención logopédica para evitar que la condición empeore. Los estudios demuestran que el logopeda trabaja principalmente en los trastornos de la deglución y de la voz. Conclusión: El logopeda es un profesional indispensable en el tratamiento de pacientes con COVID-19, especialmente pacientes sometidos a intubación orotraqueal, porque a través de su intervención terapéutica, facilita el trabajo de los demás profesionales del equipo multidisciplinar y posibilita una mejor calidad de vida del paciente.
\end{abstract}

Palabras clave: Terapia del lenguaje; Trastornos de la deglución; Afonía; COVID-19; Unidades de cuidados intensivos.

\title{
1. Introdução
}

Ao final do ano de 2019, na província de Wuhey, na China um surto desconhecido de pneumonia se iniciou, pouco tempo depois a Organização Mundial da Saúde (OMS) reconheceu e nomeou a doença como coronavírus 2019 (COVID-19) (Yuen et al., 2020). Ao final do mês Janeiro de 2020, a China notificou quase 8 mil casos confirmados de COVID-19, além disso, 82 outros casos foram registrados em mais 18 países, levando a OMS a declarar uma emergência de saúde pública de interesse nacional e posteriormente, como uma pandemia (Burki, 2020).

A COVID-19 é uma doença do trato respiratório superior, causada por uma nova cepa de coronavírus até então desconhecida, a 2019-nCoV. Os indivíduos acometidos podem manifestar desde sintomas leves (ou mesmo serem assintomáticos) até sintomas graves resultando em morte (Lovato et al., 2020). Dentre os casos de COVID-19, relatos sugerem que até $20 \%$ dos indivíduos infectados requerem hospitalização, e desses, até $25 \%$ necessitam de cuidados em unidade de terapia intensiva (UTI). Essas taxas variam de acordo com diferenças culturais em relação aos critérios de admissão à UTI e características regionais, como idade da população e prevalência de outras comorbidades. Desenvolvimento de dispneia e síndrome respiratória aguda grave são as indicações mais comuns de internação em UTI (Nascimento et al., 2021).

O desenvolvimento de complicações pós intubação são muito comuns, visto que a passagem do tubo pela orofaringe e laringe juntamente com as medicações utilizadas podem levar modificações na anatomia da glote e influenciam a atividade dos músculos relacionados à deglutição. Neste sentido, a atuação do fonoaudiólogo na equipe multiprofissional do paciente com COVID-19 é de grande importância, especialmente na reabilitação pós terapia intensiva. (Guan et al., 2020).

Ressaltando a importância deste profissional, o professor de medicina de reabilitação da Erasmus University Medical Center em Rotterdam, Holanda, elaborou um vídeo fazendo uma chamada aos fonoaudiólogos para que atuem de forma mais robusta na reabilitação dos pacientes com COVID-19 durante toda sua recuperação (Stam, 2020). Diante disso, este estudo teve como objetivo revisar a literatura disponível sobre a importância da atuação do fonoaudiólogo em pacientes com COVID19 submetidos à terapia intensiva. 


\section{Referencial Teórico}

\section{Um novo coronavírus (SARS-CoV-2) e a pandemia de COVID-19}

Os coronavírus são conhecidos pela ciência desde 1965 quando Tyrell e Bynoe o isolaram da secreção nasal de uma criança com resfriado, dando-lhes esse nome devido à sua semelhança com uma coroa solar. Desde então, várias espécies de coronavírus foram isoladas e estudadas, divididas em quatro subfamílias: alfa e beta que são originários de mamíferos e gama e delta, identificados comumente em porcos e aves ( $\mathrm{Li}$ et al., 2020).

Essa família de vírus são fitas simples de RNA que infectam humanos e alguns animais, sendo o SARS - CoV - 2 - o novo coronavírus - pertencente à subfamília de coronavírus beta. O SARS - CoV - 2 foi apontado, ao final do ano de 2019 como o responsável por um surto de pneumonia até então de etiologia desconhecida na cidade de Wuhan, na China (Xu et al., 2020).

O surto foi se espalhando cidade a cidade até atingir todos os países do mundo, levando a Organização Mundial de Saúde - OMS a reconhecer a síndrome respiratória como uma doença altamente contagiosa e de infecciosidade generalizada e declarar uma pandemia, causada pela doença do coronavírus (COVID-19). Atualmente, o número de casos confirmados em todo o mundo passa dos 169604858 e o número de mortos era de 3530837 até o final do mês de maio de 2021 (Who, 2021).

Os indivíduos acometidos com COVID-19 frequentemente apresentam sintomas como tosse, febre, congestão nasal, fadiga, dores de cabeça e sintomas gastrintestinais. Além disso, muitos pacientes apresentaram-se assintomáticos mesmo após diagnóstico positivo para a doença. Entretanto, o sinal clínico mais preocupante e que permitiu a diferenciação da doença foi a pneumonia acompanhada de falta de ar, o que tem levado muitos pacientes a ter complicações respiratórias graves exigindo a intubação orotraqueal (Guan et al., 2020; Lai et al., 2020).

Distúrbios de deglutição são muito frequentes em pacientes submetidos a longos períodos de intubação, e, portanto, é necessário reforçar a importância da avaliação e do acompanhamento desses pacientes durante sua reabilitação. Nesse sentido, o atendimento fonoaudiológico se torna indispensável pós COVID-19 (Freitas et al., 2020).

\section{Atuação do fonoaudiólogo na recuperação de pacientes com COVID-19}

A atuação da equipe multidisciplinar no paciente com COVID-19 garante maior cuidado na reabilitação do paciente, é uma estratégia muito importante diante da grande demanda por profissionais, facilitando com que o paciente tenha todas as suas necessidades supridas. Na equipe multidisciplinar, o fonoaudiólogo apresenta função de grande importância dentro da Unidade de Terapia Intensiva (UTI) identificando possíveis alterações na deglutição (Guan et al., 2020; Silva et al., 2016).

Além disso, o fonoaudiólogo deve orientar a melhor estratégia no momento da reintrodução da alimentação por via oral, de acordo com a evolução do quadro clínico do paciente, além de auxiliar durante todo o processo de reabilitação. Uma das principais preocupações que se deve ter com o paciente está relacionadas às consequências e complicações da intubação orotraqueal. Por essa doença afetar o trato respiratório, é muito comum o surgimento de disfagia (Pere, 2020).

O acompanhamento fonoaudiológico contribui evitando prejuízos as alterações respiratórias dos indivíduos com COVID-19, intervenção esta que dependerá do tipo e da gravidade da disfagia e cada caso e da estrutura com a qual o profissional conta em cada hospital. Embora a atuação do fonoaudiólogo na reabilitação pós covid-19 não seja tão reconhecida, este profissional dará um suporte técnico a todos os outros profissionais, melhorando a qualidade de vida dos pacientes (Guan et al., 2020). 
Research, Society and Development, v. 10, n. 8, e13610817154, 2021

(CC BY 4.0) | ISSN 2525-3409 | DOI: http://dx.doi.org/10.33448/rsd-v10i8.17154

Alguns estudos sugerem que existem muitos benefícios quando uma equipe multidisciplinar atua no manejo de pacientes com traqueostomia e decanulação. Neste sentido, o papel do fonoaudiólogo se concentra no controle das secreções orais com exercícios que tem como objetivo aumentar a consciência do paciente e liberação da saliva (Hashem et al., 2016).

É importante ressaltar que durante a realização dos procedimentos, os fonoaudiólogos se expõem muito aos fluídos corporais do paciente com COVID-19, portanto é importante seguir corretamente as instruções de segurança recomendadas pelas autoridades competentes da área de fonoaudiologia bem como o uso dos equipamentos de segurança adequados (Ashwini et al., 2020).

\section{Metodologia}

Trata-se de uma revisão integrativa da literatura na qual tem como finalidade reunir e sintetizar o conhecimento científico já produzido sobre o tema investigado, ou seja, permite buscar, avaliar e sintetizar as evidências disponíveis para a sua incorporação na prática (Silveira et al., 2005). Nesse sentido, o presente estudo teve que teve como pergunta norteadora: Como se dar a atuação do fonoaudiólogo na reabilitação do paciente com COVID-19? Levando em consideração os procedimentos adequados para a sua realização.

Foram incluídas pesquisas realizadas nas bases de dados Bireme, Scielo, Science Direct, e PubMed. Foram considerados os estudos publicados nos últimos 03 anos, visto que a temática escolhida teve seu foco no final do ano de 2019 e 2021. Como critério de inclusão foram considerados estudos que avaliaram o papel do fonoaudiólogo na reabilitação do paciente com COVID-19.

Como critérios de exclusão foram considerados estudos que excederam o tempo de publicação estabelecido para esta pesquisa e estudos que apenas citam o papel do fonoaudiólogo sem ter a COVID-19 como foco central do estudo.

Foram selecionados os artigos mais originais e relevantes que se encaixavam nos critérios de inclusão e exclusão do estudo, preferindo os trabalhos mais recentes. A busca de artigos foi realizada nos idiomas português e inglês com os seguintes descritores "Fonoaudiologia", “Transtornos de Deglutição", "Disfonia”, “COVID-19”, "Unidades de Terapia Intensiva”.

A estratégia de busca teve como base os descritores que foram dispostos da seguinte forma: (Fonoaudiologia OR Covid-19) AND (Transtorno de Deglutição OR Disfonia OR Covid-19) AND (Unidade de Terapia Intensiva OR Covid-19). A estratégia completa encontra-se no material suplementar (Quadro 1).

\begin{tabular}{|l|}
\hline \multicolumn{1}{|c|}{ Quadro 1 - Estratégias de busca usada em todas as bases de dados } \\
\hline $\begin{array}{l}\text { (Fonoaudiologia OR Covid-19) AND (Transtorno de Deglutição OR Disfonia OR Covid-19) AND } \\
\text { (Unidade de Terapia Intensiva OR Covid-19) }\end{array}$ \\
\hline $\begin{array}{l}\text { (Speech Therapy OR Covid-19) AND (Deglutition Disorder OR Dysphonia OR Covid-19) AND } \\
\text { (Intensive Care Unit OR Covid-19) }\end{array}$ \\
\hline
\end{tabular}

Fonte: Autores (2021).

A seleção dos artigos foi realizada seguindo a metodologia PRISMA (Preferred Reporting Items for Sistematic Reviews and Meta-analyses), como demonstra a Fluxograma 1. 
Research, Society and Development, v. 10, n. 8, e13610817154, 2021

(CC BY 4.0) | ISSN 2525-3409 | DOI: http://dx.doi.org/10.33448/rsd-v10i8.17154

Fluxograma 1 - Fluxograma dos artigos selecionados para a revisão integrativa de literatura.
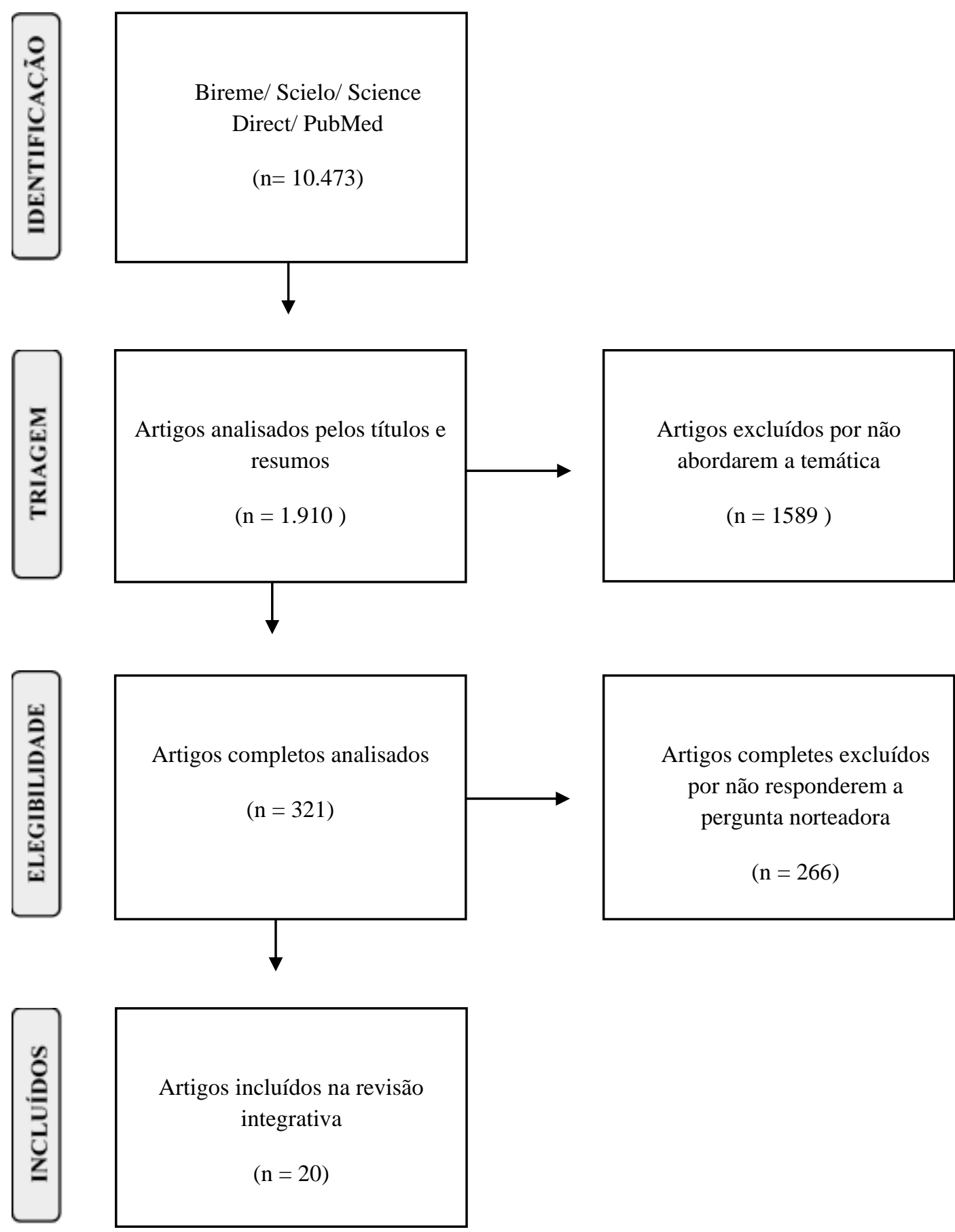

Fonte: Prisma (2009). 


\section{Resultados e Discussão}

Os pacientes acometidos pela COVID-19 provavelmente apresentarão algum tipo de comprometimento pulmonar, sobretudo nos casos graves da doença, após a recuperação. Esse comprometimento pode desencadear doenças respiratórias que estão muito ligadas à coordenação da deglutição e respiração, o que representa um risco para disfagias e broncoaspiração (Freitas et al., 2020).

Entretanto, os pacientes que apresentaram COVID-19 na forma grave, com necessidade de intubação orotraqueal não são candidatos ao acompanhamento fonoaudiológico durante o tempo em que estão em ventilação mecânica, mas não há dúvidas de que grande parte deles precisará do auxílio de um fonoaudiólogo na avaliação e tratamento dos déficits de deglutição após saírem da fase crítica (Pere, 2020).

Nesse sentido fica evidente a importância da atuação do fonoaudiólogo na reabilitação desses pacientes, dentro das limitações do profissional e da estrutura do sistema de saúde, que se encontra em um momento de crise (Guan et al., 2020). O Quadro 2 apresenta alguns estudos que ressaltam a atuação do fonoaudiólogo nesse momento de pandemia pela COVID-19.

Quadro 2 - Estudos que avaliaram a importância da atuação do fonoaudiólogo na reabilitação do paciente com COVID-19. Teresina - PI, 2020.

\begin{tabular}{|c|c|c|c|c|}
\hline Referência & População/Amostra & Objetivo & Metodologia & Resultados \\
\hline $\begin{array}{c}\text { Lima et al., } \\
2020\end{array}$ & $\begin{array}{l}\text { Pacientes com COVID- } \\
19 \text { submetidos à } \\
\text { tratamento } \\
\text { fonoaudiológico }(\mathrm{n}= \\
77)\end{array}$ & $\begin{array}{c}\text { Descrever a evolução } \\
\text { funcional da deglutição em } \\
\text { pacientes com COVID-19 } \\
\text { submetidos à intervenção } \\
\text { fonoaudiológica na Unidade } \\
\text { de Tratamento Intensivo } \\
\text { (UTI). }\end{array}$ & $\begin{array}{c}\text { Avaliação da capacidade de } \\
\text { deglutição e de recuperação da } \\
\text { deglutição através da escala para } \\
\text { avaliação de deglutição American } \\
\text { Speech-Language-Hearing } \\
\text { Association National Outcome } \\
\text { Measurement System (ASHA } \\
\text { NOMS). }\end{array}$ & $\begin{array}{l}\text { Recuperação significativa na } \\
\text { função da deglutição pré e pós } \\
\text { intervenção da equipe de } \\
\text { fonoaudiologia. }\end{array}$ \\
\hline $\begin{array}{l}\text { Mooney et } \\
\text { al., } 2020\end{array}$ & $\begin{array}{l}\text { Pacientes com COVID- } \\
19 \text { que receberam alta } \\
\text { de terapia intensiva, } \\
\text { encaminhados para } \\
\text { tratamento } \\
\text { fonoaudiológico }(n=3)\end{array}$ & $\begin{array}{c}\text { Determinar os resultados de } \\
\text { uma colaboração entre } \\
\text { fisioterapia (PT) e } \\
\text { fonoaudiologia (SLP) no } \\
\text { tratamento de pacientes } \\
\text { submetidos à colocação de } \\
\text { traqueostomia como parte } \\
\text { do tratamento para COVID- } \\
19 .\end{array}$ & $\begin{array}{c}\text { Aplicação de técnicas de } \\
\text { reorganização cognitiva, } \\
\text { comunicação verbal e não verbal, } \\
\text { controle de secreção e função da } \\
\text { deglutição de acordo com as } \\
\text { necessidades de cada paciente. }\end{array}$ & $\begin{array}{l}\text { O fonoaudiólogo foi capaz de } \\
\text { melhorar significativamente a } \\
\text { deglutição e a comunicação } \\
\text { verbal, o que por sua vez } \\
\text { melhorou a participação dos } \\
\text { pacientes no tratamento com } \\
\text { fisioterapia. }\end{array}$ \\
\hline $\begin{array}{c}\text { Ramage, } \\
2020\end{array}$ & $\begin{array}{l}\text { Ensaios controlados } \\
\text { para COVID-19 e } \\
\text { comprometimento } \\
\text { cognitivo e de } \\
\text { comunicação }\end{array}$ & $\begin{array}{l}\text { Revisar os relatos sobre } \\
\text { agravos neurológicos e de } \\
\text { comunicação no paciente } \\
\text { com COVID-19 e enfatizar } \\
\text { a importância da atuação do } \\
\text { fonoaudiólogo no } \\
\text { tratamento. }\end{array}$ & $\begin{array}{l}\text { Registros de ensaios pesquisados } \\
\text { para COVID-19 e comportamento } \\
\text { cognitivo e de comunicação pós } \\
\text { terapia intensiva. }\end{array}$ & $\begin{array}{l}\text { O autor usa o artigo para fazer } \\
\text { uma chamada aos } \\
\text { fonoaudiólogos para que atuem } \\
\text { de forma mais expressiva na } \\
\text { rotina do tratamento e terapia } \\
\text { intensiva de pacientes com } \\
\text { COVID-19, enfatizando sua } \\
\text { importância } \\
\end{array}$ \\
\hline $\begin{array}{l}\text { Portela et } \\
\text { al., } 2020\end{array}$ & $\begin{array}{l}\text { Profissionais de } \\
\text { fonoaudiologia que } \\
\text { atuavam na área }\end{array}$ & $\begin{array}{l}\text { Divulgar a atuação do } \\
\text { Fonoaudiólogo que trabalha } \\
\text { na área da disfagia no } \\
\text { período da pandemia da } \\
\text { COVID-19 }\end{array}$ & $\begin{array}{l}\text { Realização de entrevistas, } \\
\text { gravação de vídeos e obtenção de } \\
\text { imagens de fonoaudiólogos } \\
\text { atuantes no tratamento de } \\
\text { pacientes com COVID-19 }\end{array}$ & $\begin{array}{l}\text { Elaboração de um vídeo feito a } \\
\text { partir dos comentários dos } \\
\text { fonoaudiólogos sobre a atuação } \\
\text { desse profissional na disfagia em } \\
\text { pacientes com COVID-19, } \\
\text { reforçando a importância destes } \\
\text { profissionais. }\end{array}$ \\
\hline
\end{tabular}

Fonte: Dados da pesquisa.

O estudo realizado por Lima et al. (2020) evidenciou a importância da atuação do fonoaudiólogo na deglutição dos pacientes submetidos a intubação orotraqueal por consequência das complicações respiratórias da COVID-19. Um dos fatores 
que pode comprometer seriamente a deglutição do paciente com COVID-19 é a perca do olfato e do paladar, sintomas iniciais da doença. Esse fator pode se tornar um obstáculo na fase preparatória oral da deglutição e diminuir a ingestão de alimentos (Crefono, 2020).

O comprometimento do trato respiratório superior pelo coronavírus, a tosse e/ou a falta de ar que podem estar presentes, também podem contribuir para as dificuldades na deglutição. Em alguns casos, todo esse quadro pode levar o paciente à necessidade de intubação orotraqueal por longos períodos e a própria extubação pode levar ao desenvolvimento de outros distúrbios de deglutição. Esse procedimento pode resultar em lesões em todo o trato respiratório e cavidade oral, diminuindo a sensibilidade local e a motricidade, podendo levar à disfagias orofaríngeas (Ashwini et al., 2020; Sassi et al., 2018).

Nesse momento a atuação do fonoaudiólogo se torna indispensável, através da avaliação e intervenção fonoaudiológica a fim de evitar que o quadro se agrave ainda mais, evoluindo para pneumonia por aspiração e até mesmo reintubação. Quanto mais se aprende sobre o impacto dessa nova doença sobre a deglutição e a comunicação, mais importante se torna o fonoaudiólogo (Freitas et al., 2020). Diante disso, o resultado evidenciado por Mooney et al. (2020) mostra que melhorando a capacidade de deglutição do paciente, contribui-se com a adesão do mesmo a outras intervenções da equipe multidisciplinar.

Além disso, estes mesmos autores observaram que a intervenção fonoaudiológica foi capaz de melhorar a comunicação verbal dos pacientes com COVID-19. Mesmo após a alta hospitalar, o paciente recuperado de COVID-19 pode apresentar alterações na comunicação e na qualidade vocal como sequela da doença, além de dificuldades na coordenação respiratória, exigindo intervenção fonoaudiológica competente para reabilitação específica em cada caso (Conselho federal de fonoaudiologia, 2020).

Estudo recente realizado por Lechien et al. (2020) verificou a ocorrência de disfonia, ou seja, dificuldade na produção da voz em alguns pacientes que testaram positivo para a COVID-19. Além disso, alguns dos pacientes também relataram perda parcial ou total da voz. Nesse estudo, foram avaliados mais de 700 pacientes e cerca de $20 \%$ destes relataram os sintomas disfônicos. Neste estudo, os autores encontraram uma associação significativa entre a gravidade da disfonia e a gravidade da disfagia, além de associação positiva desta com tosse.

A terapia fonoaudiológica direcionada ao tratamento de disfonia consiste na diminuição da tensão da musculatura fonatória e respiratória, melhorando a habilidade vocal e direcionando o paciente a desenvolver um padrão de voz com fonação mais relaxada (Buselli et al., 2020). Diante da importância da atuação do fonoaudiólogo na comunicação verbal do paciente com COVID-19, Ramage (2020) utilizou um estudo científico sobre agravos neurológicos e de comunicação para fazer uma chamada a estes profissionais para que intensifiquem os cuidados com estes pacientes precocemente, não se limitando ao momento pós terapia intensiva.

Portela et al. (2020) entrevistaram fonoaudiólogos que estavam atuando diretamente no tratamento de pacientes acometidos por COVID-19 e elaboraram um vídeo onde os profissionais que participaram da pesquisa enfatizam as dificuldades encontradas no contexto da pandemia pelo novo coronavírus, relacionadas à nova paramentação e uso de equipamentos de segurança individual, treinamentos e orientações recebidas.

Dessa forma fica claro que o fonoaudiólogo é profissional indispensável na equipe multidisciplinar durante a pandemia de COVID-19 (e após), embora sua atuação seja ainda pouco difundida, é um profissional fundamental no tratamento da deglutição e outras complicações, dando suporte a todo o restante da equipe. O grande objetivo da fonoaudiologia nesse contexto, baseia-se em oferecer mais qualidade de vida aos pacientes após a alta (Porto et al., 2020). 
Research, Society and Development, v. 10, n. 8, e13610817154, 2021

(CC BY 4.0) | ISSN 2525-3409 | DOI: http://dx.doi.org/10.33448/rsd-v10i8.17154

\section{Considerações Finais}

Diante do exposto, fica evidente que o fonoaudiólogo é um profissional indispensável no tratamento de pacientes com COVID-19, sobretudo os pacientes submetidos à intubação orotraqueal, pois através da sua intervenção terapêutica, facilita o trabalho dos demais profissionais da equipe multidisciplinar e possibilita mais qualidade de vida ao paciente.

É importante ressaltar que os profissionais de fonoaudiologia devem estar sempre atualizados e atentos às orientações e diretrizes das autoridades de saúde, tanto para evitar a contaminação pelo novo coronavírus como para evitar complicações no quadro clínico do paciente.

Destaca-se que ainda são poucos os estudos que buscam evidenciar o quanto é importante que os pacientes com COVID-19 tenham acesso à intervenção fonoaudiológica, sugere-se que mais estudos sejam realizados nesse sentido, tanto para evidenciar a importância desse profissional como para auxiliar com informações científicas que facilitem a reabilitação destes pacientes.

\section{Referências}

Ashwini, M., Namasivayam-MacDonail, E. L. \& Riquelme, 1. F. (2020). Speech therapy management for adults with COVID-19 in the acute hospital setting: initial recommendations to guide clinical practice. American Journal of Speech-Language Pathology, Canada. 29(4), 1850-65. 10.1044 / 2020_AJSLP-2000096

Burki, T. K. (2020). Coronavirus in China. The Lancet. 8(3), 238. https://doi.org/10.1016/S2213-2600(20)30056-4

Buselli, R., Corsi, M., Necciari, G., Pistolesi, P., Baldanzi, S., Chiumiento, M., Del Lupo, E., Guerra, P. D. \& Cristaudo, A. (2020). Sudden and persistent dysphonia within the framework of COVID-19. The case report of a nurse, Pisa, Italy. 9(1), 1-4. 10.1016/j.bbih.2020.100160

Conselho federal de fonoaudiologia - Crefono. (2020). Recomendação CFFa $n^{o}$ 19. https://www.fonoaudiologia.org.br/cffa/

Freitas, A. S., Zica, G. M. \& Albuquerque, C. L. (2020). Pandemia de coronavírus (COVID-19): o que os fonoaudiólogos devem saber. CoDAS, Brasil. 32(3), 22. http://dx.doi.org/10.1590/2317-1782/20192020073

Guan, W., et al. (2020). Clinical Characteristics of Coronavirus Disease 2019 in China. New England Journal of Medicine, Massachusetts, Boston. 382(18), 1708-20. 10.1056/NEJMoa2002032

Hashem, M. D., Nelliot, A. \& Needham, D. M. (2016). Early mobilization and rehabilitation in the ICU: Moving Back to the Future. Respiratory Care, Tampa, Florida. 61(7), 971-79. 10.4187 / respcare.04741.

Lai, C. C., Shih, T. P., Ko, W. C., Tang, H. J. \& Hsueh, P. R. (2020). Severe acute respiratory syndrome coronavirus 2 (SARS-CoV-2) and corona virus disease-2019 (COVID-19): the epidemic and the challenges. International Journal of Antimicrobial Agents, Taiwan. 55(03), 105924. 10.1016/j.ijantimicag.2020.105924

Lechien, C. S., Jerome, R., Chiesa-Estomba, M., et al. (2020). Features of Mild-to-Moderate COVID-19 Patients with Dysphonia. Journal of voice. Journal Official da Voice Foundation, Paris, France, S0892-1997(20), 30183-191. 10.1016/j.jvoice.2020.05.012

Li, H., Liu, S. M., Yu, X. H., Tang, S.L. \& Tang, C. K. (2020). Coronavirus disease 2019 (COVID-19): current situation and future perspectives. International Journal Antimicrob Agents. 55(5), 105951. 10.1016/j.ijantimicag.2020.105951

Lima, M. S. De., Sassi, F. C., Medeiros, G. C. De., Ritto, A. P. \& Andrade, C. R. F. De. (2020). Evolução funcional da deglutição em pacientes com COVID19 internados em UTI. CoDAS, São Paulo, Brasil. 32(4), 1-3. https://doi.org/10.1590/2317-1782/20192020222

Lovato, A., Filippis, C. De. \& Marioni, G. (2020). Upper airway symptoms in coronavirus disease 2019 (COVID-19). American Journal of Otolaryngology, Padova, Italy. 41(3), 102474. 10.1016 / j.amjoto.2020.102474

Mooney, B., Lawrence, C., Johnson, E. G., Slaboden, A. \& Ball, K. (2020). How COVID-19 patients were motivated to speak: a series of interdisciplinary rehabilitation cases. HSS Journal, New York. 16(1), S56-S63. 10.1007/s11420-020-09778-0

Nascimento, P. H. J., et al. (2021). COVID-19 e Injúria Miocárdica em UTI Brasileira: Alta Incidência e Maior Risco de Mortalidade IntraHospitalar. Arquivos Brasileiros de Cardiologia, 116(2), 275-82. https://www.scielo.br/j/abc/a/Txj6w8qYFn9D9nWsXTFcncw/abstract/?lang=pt

Pere, C. (2020). Oropharyngeal Dysphagia in Patients With COVID-19 Apr. 14, 2020. ClinicalTrials.gov., Espanha, 2020. https://clinicaltrials.gov/ct2/show/NCT04346212

Portela, M. L. Dos S., Cruz, Y. A., Cruz, E. R., et al. (2020) Atuação fonoaudiológica em disfagia em tempos da Pandemia da Covid-19. Mostra de Inovação e Tecnologia - São Lucas, 1(1), 1-2. https://doi.org/10.1590/0100-6991e-20181687 
Research, Society and Development, v. 10, n. 8, e13610817154, 2021

(CC BY 4.0) | ISSN 2525-3409 | DOI: http://dx.doi.org/10.33448/rsd-v10i8.17154

Porto, A. C. L., Oliveira, L. B., Cabral, J. de A., et al. (2020) Atuação Fonoaudiológica em pacientes COVID-19: Revisão Integrativa. Cadernos esp. Ceará, Fortaleza, Brasil. 14(1), 38-44. https://cadernos.esp.ce.gov.br/index.php/cadernos/article/view/305

Ramage, A. E. (2020). Potential for Cognitive Communication Impairment in COVID-19 Survivors: A Call to Action for Speech-Language Pathologists. American Journal of Speech-Language Pathology, Inglaterra. 29(12), 1821-32. https://doi.org/10.1044/2020_AJSLP-20-00147

Sassi, F. C., Medeiros, G. C. De., Zambon, L. S., Zilberstein, B. \& Andrade, C. R. F. (2018). Avaliação e classificação da disfagia pós-extubação em pacientes críticos. Revista do Colégio Brasileiro Cirurgiões, Rio de Janeiro, Brasil. 45(3), 1687. https://doi.org/10.1590/0100-6991e-20181687

Silva, D. L. R., Lira, F. O. Q., Oliveira, J. C. C. \& Canuto, M. S. B. (2016) Atuação da fonoaudiologia em unidade de terapia intensiva de um hospital de doenças infecciosas de Alagoas. Revista CEFAC, Maceió, Brasil. 18(1), 174-83. 10.1590/1982-0216201618112015

Silveira, R. C. C.P. \& Galvão, C. M. (2005) O cuidado de enfermagem e o cateter de Hickman: a busca de evidências [dissertation]. Ribeirão Preto: Universidade de São Paulo, Escola de Enfermagem de Ribeirão Preto. https://www.scielo.br/j/ape/a/KBW9WsfzTKZh6DKgY SNDPYq/?format=pdf\&lang=pt

Stam, H. J., Stucki, G. \& Bickenbach, J. (2020). Covid-19 and Post Intensive Care Syndrome: A Call for Action. J Rehabil Med, Holland. 52(4), 44 . 10.2340 / $16501977-2677$

WHO - World Health Organization. (2021). Atualização operacional semanal no COVID-19. https://www.who.int/publications/m/item/weeklyepidemiological-update-on-covid-19---1-june-2021

Xu. X., Chen, P., Wang, J., et al. (2020). Evolution of the novel coronavirus from the ongoing Wuhan outbreak and modeling of its spike protein for risk of human transmission. Sci China Life Sci, China. 63(3), 457-60. 10.1007/s11427-020-1637-5

Yuen, K., Ye, Z., Fung, S., et al. (2020). SARS-CoV-2 and COVID-19: The most important research questions. Cell Biosci., 10(1), 40. https://doi.org/10.1186/s13578-020-00404-4 